\title{
Article \\ Objective and Subjective Prevalence of Obstructive Sleep Apnoea/Hypopnoea Syndrome in UK Adults with Down Syndrome: A Strong Marker for Diurnal Behavioural Disturbances
}

\author{
Elizabeth A. Hill ${ }^{1}{ }^{\mathbb{D}}$, Linda J. Williams ${ }^{2} \mathbb{D}$, Sally-Ann Cooper $^{3}\left(\mathbb{C}\right.$ and Renata L. Riha ${ }^{1,4, *,+}$ \\ 1 Sleep Research Unit, Centre for Clinical Brain Sciences, The University of Edinburgh, \\ Edinburgh EH16 4SB, UK; lizzie.hill@ndcn.ox.ac.uk \\ 2 Edinburgh Clinical Trials Unit, The Usher Institute, The University of Edinburgh, Edinburgh EH16 4UX, UK; \\ linda.williams@ed.ac.uk \\ 3 Institute of Health and Wellbeing, University of Glasgow, 1st Floor Administrative Building, \\ Gartnavel Royal Hospital, Glasgow G12 0XH, UK; sally-ann.cooper@glasgow.ac.uk \\ 4 Department of Sleep Medicine, Royal Infirmary of Edinburgh, Edinburgh EH16 4SA, UK \\ * Correspondence: rriha1@exseed.ed.ac.uk; Tel.: +44-131-242-3872 \\ $+\quad$ The corresponding author attests that all listed authors meet authorship criteria and that no others meeting \\ the criteria have been omitted.
}

check for

updates

Citation: Hill, E.A.; Williams, L.J.; Cooper, S.-A.; Riha, R.L. Objective and Subjective Prevalence of Obstructive Sleep Apnoea/ Hypopnoea Syndrome in UK Adults with Down Syndrome: A Strong Marker for Diurnal Behavioural Disturbances. Brain Sci. 2021, 11, 1160. https://doi.org/10.3390/

brainsci11091160

Academic Editor: Roumen Kirov

Received: 19 May 2021

Accepted: 23 August 2021

Published: 31 August 2021

Publisher's Note: MDPI stays neutral with regard to jurisdictional claims in published maps and institutional affiliations.

Copyright: (c) 2021 by the authors. Licensee MDPI, Basel, Switzerland. This article is an open access article distributed under the terms and conditions of the Creative Commons Attribution (CC BY) license (https:/ / creativecommons.org/licenses/by/ $4.0 /)$.

\begin{abstract}
Prior to this study, the prevalence of obstructive sleep apnoea/hypopnoea syndrome (OSAHS) in adults with Down syndrome was unknown. We hypothesized that unrecognised OSAHS could have an additional deleterious impact on mood and behavioural disturbances in this group of people. Aims: To assess the prevalence of OSAHS in adults with Down syndrome in the United Kingdom, subjectively and objectively, and ascertain its association with diurnal behavioural disturbances. Method: Cross-sectional questionnaire study with home sleep apnoea testing (HSAT) during 2011-2015 across the four nations of the United Kingdom. Participants were adults aged $\geq 16$ years with Down syndrome. Main outcome measures were: self- or caregiver-completed questionnaire data, including the Pictorial Epworth Sleepiness Scale (pESS), selected domains of the Developmental Behavioural Checklist for Adults (DBC-A), anthropometric measures, and symptoms of OSAHS. Objective prevalence was undertaken in a sample of responders using HSAT. Results: Responses were received from 1321/5270 participants (25\%), with 1105 valid responses (21\%). Eightyone participants $(7 \%)$ reported a prior diagnosis of OSA, of whom 38 were receiving therapy. Using validated algorithms, a diagnosis of OSAHS was probable in 366 participants (35\%), who were younger, with higher BMI and higher mean total pESS $(p<0.0001)$. A total of $23 \%$ of participants had a pESS > 10. OSAHS was a strong marker for behavioural disturbances on the DBC-A depression, disruption and anti-social subscales $(p<0.001)$. Of 149 individuals who underwent HSAT, 42\% were diagnosed with OSAHS. Conclusions: Untreated OSAHS in Down syndrome is common and associated with behavioural and mood disturbances. Improving awareness of OSAHS amongst adults with Down syndrome, their families and healthcare professionals is essential.
\end{abstract}

Keywords: sleep-related breathing disorders; obstructive sleep apnoea; home sleep apnoea testing; excessive daytime sleepiness; down syndrome; trisomy 21; prevalence

\section{Introduction}

Down syndrome, present in 1 in 1000 live births in Europe [1], is the commonest form of intellectual disability worldwide. Currently, $>37,000$ people have Down syndrome in England and Wales alone [2]. Life expectancy in people with DS is rising, and may exceed 50 years [3].

Sleep-disordered breathing is characterised by repetitive pauses in breathing during sleep, and affects approximately $24 \%$ of the general adult population [4]. Obstructive sleep 
apnoea/hypopnoea syndrome (OSAHS) is diagnosed when nocturnal apnoea results in significant diurnal symptoms, including excessive daytime somnolence, impaired cognitive function, reduced quality of life, and behavioural and emotional disturbances [4]. The adult prevalence of OSAHS is $2 \%$ in women and $4 \%$ in men [4]. OSAHS is an independent risk factor for cardiovascular morbidity and mortality, including hypertension, myocardial infarction and stroke [4].

The Down syndrome phenotype includes a flattened face, short neck, generalised hypotonia, loose ligaments, and a tendency towards weight gain-all risk factors for OSAHS. The additional impact of OSAHS on cognitive ability in DS is still unknown, but may have additional deleterious effects [5].

OSAHS prevalence in children with Down syndrome has been reported to be approximately 45-55\% [6]; the prevalence in adults with Down syndrome was unknown. Two small studies in adults with Down syndrome $(n=6 ; n=16)$ reported $>80 \%$ of their respective samples to have obstructive sleep apnoea, but these studies may not be representative of the wider Down syndrome population [7,8]. A questionnaire study in Canada [9] reported a diagnosis of OSA in 21\% of 223 individuals with Down syndrome aged 1 month to $>40$ years, but prevalence data for adults only were not reported.

The aim of this study was to establish the prevalence and severity of symptoms of OSAHS and related behavioural and emotional disturbances in adults with Down syndrome using subjective and objective measures. We hypothesised that OSAHS would be a strong marker for diurnal behavioural disturbances in adults with Down syndrome.

\section{Methods}

\subsection{Study Design and Data}

The study was approved by the Scotland A Research Ethics Committee (REC no: 11/MRE00/3). Return of a completed questionnaire was considered implicit consent to participate. Individuals undertaking home sleep apnoea testing gave written informed consent.

\subsection{Subjective Prevalence}

Questionnaires and pre-paid reply envelopes were sent to 5266 UK-based adults ( $\geq 16$ years) with Down syndrome and their caregivers between 2011 and 2014. Potential study participants were identified by local and national organisations supporting people with Down syndrome (see Acknowledgements).

The questionnaire comprised a section for completion by the individual with Down syndrome and a section for completion by a relative/caregiver. Anthropometric, comorbidity, medication, demographic, and sleep disturbance data (including frequency per week of snoring, witnessed apnoeas, nocturnal choking episodes, frequent awakenings, unrefreshing sleep, and daytime sleepiness) were collected. The pictorial version of the Epworth Sleepiness Scale (pESS) [10], designed to enhance understanding and accessibility in a broader adult population, was also administered. Prior to this study, the pESS had not been used in a population with Down syndrome.

Additionally, caregivers completed three subscales of the Developmental Behaviour Checklist for Adults (DBC-A) [11], which may be related to sleep deprivation: Disruptive, Anxiety/Antisocial, and Depressive. Subscales were scored as described by Taffe et al. [12]. The DBC-A has been used extensively in populations with people with Down syndrome.

Medications were classified using the World Health Organisation Anatomical Therapeutic Chemical Classification System [13].

Reported symptoms suggestive of OSAHS were defined using three previously validated algorithms: [14]

1. Snoring $\geq 3$ nights / week plus (witnessed apnoeas, or pESS $>10$ ).

2. Snoring $\geq 3$ nights/week plus (witnessed apnoeas, or unrefreshing sleep $\geq 3$ nights/week).

3. Snoring $\geq 3$ nights/week plus (witnessed apnoeas, or daytime sleepiness $\geq 3$ /week). 


\subsection{Objective Prevalence}

In order to assess objective prevalence of OSAHS by sampling as true a cross-section of the target population as possible, responders were considered eligible if aged $\geq 16$ years with Down syndrome and not currently using CPAP; home sleep apnoea testing was offered regardless of subjective sleepiness, sleep-related symptoms, previous sleep diagnoses or any other variables reported in the questionnaire and first recruitment occurred 10 January 2012.

Home sleep studies were conducted using the Embletta ${ }^{\circledR}$ Gold $^{\mathrm{TM}}$ (Embla Systems LLC., Amsterdam, The Netherlands) cardiorespiratory polygraphy device. This is a level III device [15], with the capacity to record multiple channels of physiological data. Home sleep apnoea testing by level III polygraphy is routinely used in clinical practice across the UK. Channels were recorded in broad accordance with AASM guidelines for full PSG [16], as recommended by the AASM guidelines for portable monitoring [15], and included nasal airflow and snoring via nasal pressure cannula, respiratory effort via thoracic and abdominal respiratory inductance plethysmography bands, $\mathrm{SpO}_{2}$ via pulse oximetry and body position via an inbuilt position sensor.

All studies were manually validated and scored by one of two experienced Registered Polysomnographic Technologists using standard software (Embla ${ }^{\circledR}$ RemLogic ${ }^{\mathrm{TM}}$ Embla Systems LLC., Amsterdam, The Netherlands) in broad accordance with current international guidelines [16]. To ensure consistency of scoring, inter- and intra-rater reliability scoring was conducted in randomly selected subsets of $10 \%$ of valid studies.

\subsection{Statistical Analysis}

Standard statistical analyses were undertaken using IBM SPSS Statistics for Windows Version 19.0 (IBM Corp., Armonk, NY, USA).

All variables were checked for normality. Descriptive statistics were generated to report frequencies of sleep problems and participant characteristics. The chi-square test was used for discrete variables, Student's $t$-test for continuous variables, and Mann-Whitney U test for non-parametric data, to investigate anthropometric, sleep, and behavioural and emotional associations with probable OSA, and gender differences in sleep and behavioural and emotional characteristics. A conservative cut-off for significance was taken at $p<0.001$ to account for the effects of multiple testing (with alpha $=0.004$ to 0.0025 depending on the number of variables). Though a little stringent, we believed that this would lead to mitigating Type I error further in a sample of this size without having to undertake a Bonferroni or Holm correction on each occasion. Pearson's and Spearman's rank correlations were used to examine possible associations between anthropometrics, sleep characteristics and behavioural and emotional disturbances. Both binary logistic regression analysis (for exploring a pESS cut-off score below and above 10/24) and generalised linear modelling were undertaken to explore independent associations of OSAHS with anthropometric characteristics, medication use and behaviour/emotion. All analyses were two-tailed.

Results are presented as mean \pm standard deviation for parametric variables or median with interquartile range (IQR 25 to $75 \%$ ) for non-parametric data, or as number and percentage.

\subsection{Role of Funding Bodies and Study Sponsors}

The Chief Scientist Office (Edinburgh, UK), Baily Thomas Charitable Trust (Luton, UK) and Fondation Jérôme Lejeune (Paris, France) funded this study. ResMed (UK) Ltd. provided non-financial support via loan of home sleep study equipment. None of these parties were actively involved in the design, analysis or reporting of the study. As study sponsors, Lothian Universities Health Trust and the University of Edinburgh did not play any role in the planning, conducting or analysis of this study. 


\subsection{Public and Patient Involvement}

Adults with Down syndrome reviewed the study materials for accessibility via focus groups organised through Down's Syndrome Scotland, a national organisation supporting people with Down syndrome and their families, with amendments made in line with feedback received.

\section{Results}

Responses were received from 1321/5270 participants (25\%), of which 1105 responses (21\%) were valid for analysis (Figure 1 ).

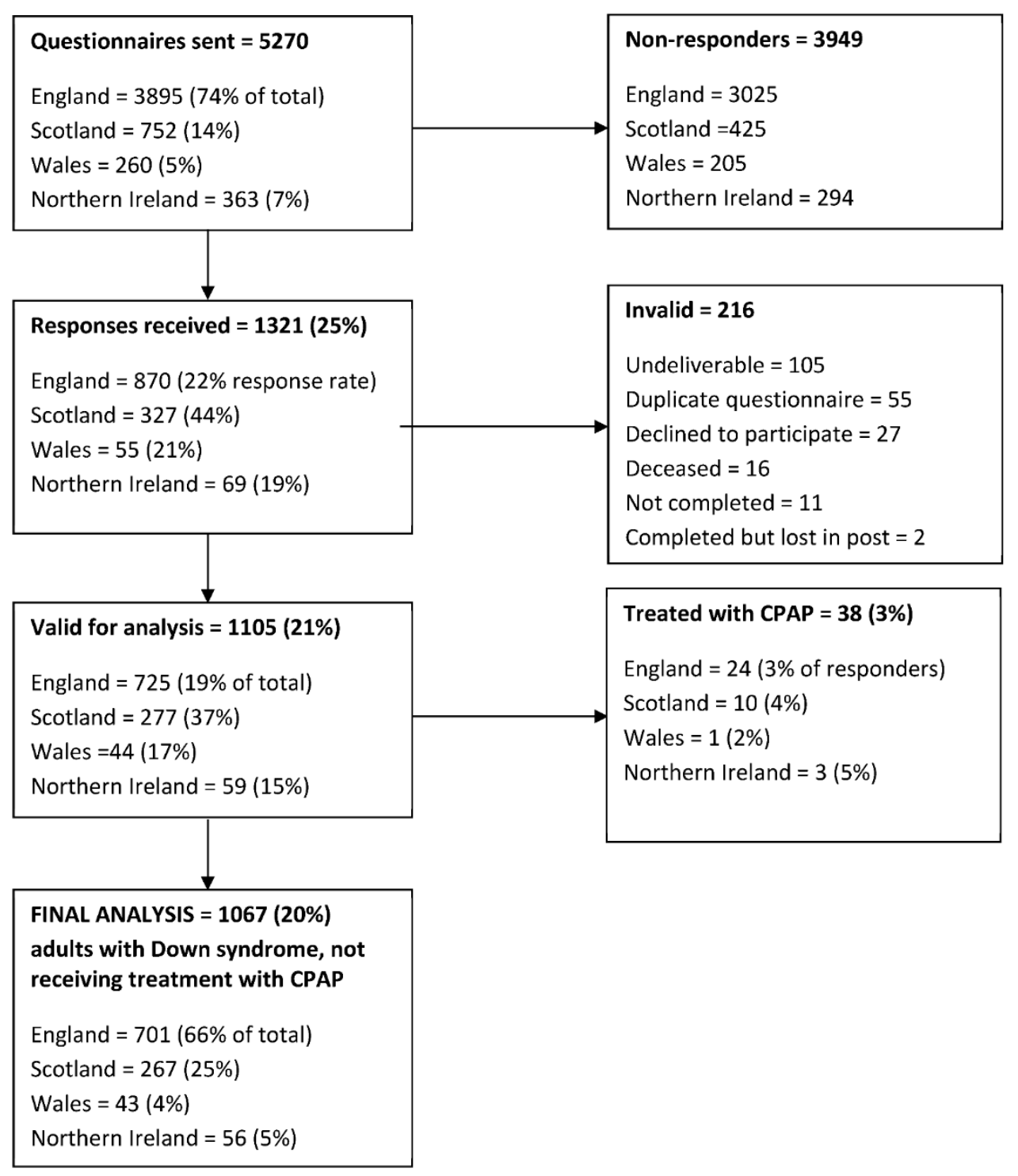

Figure 1. Summary of questionnaires distributed to adults with Down syndrome residing within the $\mathrm{UK}$, returned to the investigators and included in the final analysis.

Fifty-three men and 28 women $(7 \%)$ reported a prior diagnosis of OSA. Of these, 38 (3.4\% of the total) were receiving continuous positive airway pressure (CPAP) therapy and were excluded from further analysis. Other reported sleep disorders included insomnia $(n=2)$, narcolepsy $(n=1)$, behavioural sleep problems $(n=1)$, parasomnia $(n=1)$, headbanging $(n=1)$, myoclonic jerks $(n=1)$, nightmares $(n=1)$ and somniloquy $(n=1)$. 


\subsection{Anthropometric Data and Comorbidities}

Anthropometric characteristics of the responders included in the study are shown in Table 1. Most participants were overweight (34\%) or obese (40\%) [17].

Table 1. Anthropometric characteristics of all valid questionnaire responders, with responders on CPAP therapy excluded. Chi-square test used for parametric categorical variables, $t$-test for continuous categorical variables and Mann-Whitney $\mathrm{U}$ test for non-parametric variables. Values presented as mean $\pm \mathrm{SD}$ or $n \%$ unless otherwise stated.

\begin{tabular}{|c|c|c|c|c|c|c|c|c|}
\hline Characteristics & $\begin{array}{c}\text { Total } \\
\text { Responses }\end{array}$ & \multicolumn{2}{|c|}{$\begin{array}{l}\text { All Responders } \\
n=1067\end{array}$} & \multicolumn{2}{|c|}{$\begin{array}{c}\text { Male } \\
n=585(54.8 \%) *\end{array}$} & \multicolumn{2}{|c|}{$\begin{array}{c}\text { Female } \\
n=480(45.0 \%)\end{array}$} & $p^{* *}$ \\
\hline Age (years) & 1062 & \multicolumn{2}{|c|}{$28 \pm 9$} & \multicolumn{2}{|c|}{$28 \pm 9$} & \multicolumn{2}{|c|}{$28 \pm 9$} & 0.99 \\
\hline Body Mass Index $\left(\mathrm{kg} / \mathrm{m}^{2}\right)^{* * *}$ & 911 & \multicolumn{2}{|c|}{$29.0 \pm 6.8$} & \multicolumn{2}{|c|}{$28.2 \pm 6.6$} & \multicolumn{2}{|c|}{$30.0 \pm 6.8$} & $<0.0001$ \\
\hline Underweight $\left(<18.5 \mathrm{~kg} / \mathrm{m}^{2}\right)$ & \multirow{6}{*}{744} & 6 & $0.8 \%$ & 3 & $0.7 \%$ & 3 & $0.9 \%$ & \multirow{6}{*}{0.001} \\
\hline Normal weight $\left(18.5-24.99 \mathrm{~kg} / \mathrm{m}^{2}\right)$ & & 187 & $25.1 \%$ & 124 & $30.1 \%$ & 63 & $19.0 \%$ & \\
\hline Pre-obesity $\left(25.0-29.99 \mathrm{~kg} / \mathrm{m}^{2}\right)$ & & 255 & $34.3 \%$ & 147 & $35.7 \%$ & 107 & $32.3 \%$ & \\
\hline Obesity class I (30.0-34.99 kg/m²) & & 154 & $20.7 \%$ & 74 & $18.0 \%$ & 80 & $24.2 \%$ & \\
\hline Obesity class II $\left(35.0-39.99 \mathrm{~kg} / \mathrm{m}^{2}\right)$ & & 91 & $12.2 \%$ & 43 & $10.4 \%$ & 48 & $14.5 \%$ & \\
\hline Obesity class III $\left(\geq 40.00 \mathrm{~kg} / \mathrm{m}^{2}\right)$ & & 51 & $6.9 \%$ & 21 & $5.1 \%$ & 30 & $9.1 \%$ & \\
\hline Collar size $(\mathrm{cm})$ & 579 & \multicolumn{2}{|c|}{$40.4 \pm 4.3$} & \multicolumn{2}{|c|}{$41.3 \pm 3.8$} & \multicolumn{2}{|c|}{$38.2 \pm 4.5$} & $<0.0001$ \\
\hline \multicolumn{9}{|c|}{ Smoking status: } \\
\hline Smoker & \multirow{3}{*}{1017} & 1 & $0.1 \%$ & 1 & $0.2 \%$ & 0 & $0.0 \%$ & \multirow{3}{*}{0.54} \\
\hline Ex-smoker & & 5 & $0.5 \%$ & 2 & $0.4 \%$ & 3 & $0.6 \%$ & \\
\hline Non-smoker & & 1011 & $99.4 \%$ & 548 & $99.5 \%$ & 462 & $99.4 \%$ & \\
\hline Any medication & 1067 & 728 & $68.2 \%$ & 369 & $63.1 \%$ & 359 & $74.8 \%$ & $<0.0001$ \\
\hline \multicolumn{9}{|c|}{ Comorbidities: } \\
\hline Asthma & 1067 & 135 & $12.7 \%$ & 75 & $12.8 \%$ & 60 & $12.5 \%$ & 0.93 \\
\hline Adenoid and/or tonsil surgery & 1067 & 249 & $23.3 \%$ & 136 & $23.2 \%$ & 113 & $23.5 \%$ & 0.94 \\
\hline Stroke & 1067 & 16 & $1.5 \%$ & 7 & $1.2 \%$ & 9 & $1.9 \%$ & 0.45 \\
\hline Broken nose & 1067 & 8 & $0.7 \%$ & 6 & $1.0 \%$ & 2 & $0.4 \%$ & 0.31 \\
\hline Diabetes & 1067 & 29 & $2.7 \%$ & 13 & $2.2 \%$ & 16 & $3.3 \%$ & 0.34 \\
\hline Heart problems & 1067 & 37.2 & $3.5 \%$ & 182 & $31.1 \%$ & 215 & $44.8 \%$ & $<0.0001$ \\
\hline Hay fever & 1067 & 193 & $18.1 \%$ & 104 & $17.8 \%$ & 89 & $18.5 \%$ & 0.75 \\
\hline Thyroid problems & 1067 & 379 & $35.5 \%$ & 168 & $28.7 \%$ & 210 & $43.8 \%$ & $<0.0001$ \\
\hline Epilepsy & 1067 & 62 & $5.8 \%$ & 31 & $5.3 \%$ & 31 & $6.5 \%$ & 0.43 \\
\hline Liver problems & 1067 & 17 & $1.6 \%$ & 13 & $2.2 \%$ & 4 & $0.8 \%$ & 0.09 \\
\hline Hypertension & 1067 & 19 & $1.8 \%$ & 9 & $1.5 \%$ & 10 & $2.1 \%$ & 0.64 \\
\hline Nasal surgery & 1067 & 15 & $1.4 \%$ & 8 & $1.4 \%$ & 7 & $1.5 \%$ & 1.00 \\
\hline Kidney problems & 1067 & 23 & $2.2 \%$ & 11 & $1.9 \%$ & 12 & $2.5 \%$ & 0.53 \\
\hline Gluten intolerance & 1067 & 61 & $5.7 \%$ & 26 & $4.4 \%$ & 35 & $7.3 \%$ & 0.06 \\
\hline
\end{tabular}

${ }^{*}$ Gender of 2 responders unknown; ${ }^{* *}$ Difference between males and females; ${ }^{* * *}$ WHO BMI category calculated for participants aged $\geq 20$ years only.

Females had a significantly higher body mass index than males (males $28.2 \pm 6.6 \mathrm{~kg} / \mathrm{m}^{2}$, females $30.0 \pm 6.8 \mathrm{~kg} / \mathrm{m}^{2} ; p<0.0001$ ), but smaller collar size (males $41.3 \pm 3.8 \mathrm{~cm}$, females $38.2 \pm 4.5 \mathrm{~cm} ; p<0.0001)$. No other significant gender differences were observed.

The prevalence of comorbidities that can be related to OSAHS or potentially affecting sleep (Table 1) was low: hay fever $(18 \%)$; asthma (13\%); epilepsy (6\%); diabetes $(3 \%)$; hypertension ( $2 \%)$; stroke $(2 \%)$.

Adenotonsillar hypertrophy is known to affect upper airway size and function in both adults and children in the general population. A total of 243 responders $(23 \%)$ reported removal of tonsils and/or adenoids and $124(12 \%)$ reported adenotonsillectomy. There was a trend for those who had undergone surgery to be more likely to meet the criteria for probable OSAHS ( $42 \%$ vs. $32 \%, p=0.002$; data not shown). 


\subsection{Sleep Symptoms}

Probable OSAHS criteria [16] were met by 366 participants (35\%), who were significantly younger, with higher body mass index, higher mean total pESS and more likely to have a pESS $>10$ (all $p<0.0001$ ) (Tables 2 and 3 online). Mean pESS scores were within the normal range $(7 \pm 5)$, with no significant gender difference (Table 4 online). Excessive daytime somnolence (pESS > 10) was classified in $23 \%$ of participants, with no significant gender difference.

Table 2. Sleep and behaviour characteristics of responders meeting criteria for probable OSA on $\geq 1$ algorithm, with responders on CPAP therapy excluded. Chi-square test used for parametric categorical variables, $t$-test for continuous categorical variables and Mann-Whitney U test for non-parametric variables. Values presented as mean $\pm S D$, median (IQR) or $n \%$ unless otherwise stated.

\begin{tabular}{|c|c|c|c|c|c|c|}
\hline \multirow{2}{*}{$\begin{array}{c}\text { Characteristics } \\
\text { Prior diagnosis of obstructive sleep apnoea (OSA) }\end{array}$} & \multirow{2}{*}{$\begin{array}{c}\text { Total Responses } \\
1039\end{array}$} & \multicolumn{2}{|c|}{$\begin{array}{c}\text { Probable OSA } \\
n=366(34.3 \%) *\end{array}$} & \multicolumn{2}{|c|}{$\begin{array}{c}\text { OSA Not Suspected } \\
n=673(63.1 \%)^{*}\end{array}$} & \multirow{2}{*}{$\begin{array}{c}p \\
<0.0001\end{array}$} \\
\hline & & 31 & $8.5 \%$ & 13 & $1.9 \%$ & \\
\hline DBC-A Disruptive subscale (scale range 0-34) & 1023 & \multicolumn{2}{|c|}{$7(3-12)$} & \multicolumn{2}{|c|}{$4(1-8)$} & $<0.0001$ \\
\hline Mean item score (possible score $0-2$ ) & 1023 & \multicolumn{2}{|c|}{$0.42(0.18-0.71)$} & \multicolumn{2}{|c|}{$0.24(0.06-0.50)$} & $<0.0001$ \\
\hline Proportion of items checked (possible score $0-1$ ) & 1023 & \multicolumn{2}{|c|}{$0.41(0.18-0.59)$} & \multicolumn{2}{|c|}{$0.24(0.06-0.41)$} & $<0.0001$ \\
\hline Intensity index (possible score $0-1$ ) & 895 & \multicolumn{2}{|c|}{$0.10(0.00-0.33)$} & \multicolumn{2}{|c|}{$0.00(0.00-0.22)$} & $<0.0001$ \\
\hline DBC-A Anxiety/Antisocial subscale (scale range -2-14) & 1021 & \multicolumn{2}{|c|}{$0(-1-2)$} & \multicolumn{2}{|c|}{$0(0-1)$} & 0.047 \\
\hline Mean item score (possible score $0-2$ ) & 1021 & \multicolumn{2}{|c|}{$0.22(0.11-0.44)$} & \multicolumn{2}{|c|}{$0.11(0.00-0.33)$} & $<0.0001$ \\
\hline Proportion of items checked (possible score $0-1$ ) & 1021 & \multicolumn{2}{|c|}{$0.22(0.11-0.33)$} & \multicolumn{2}{|c|}{$0.11(0.00-0.22)$} & $<0.0001$ \\
\hline Intensity index (possible score $0-1$ ) & 766 & \multicolumn{2}{|c|}{$0.25(0.00-0.50)$} & \multicolumn{2}{|c|}{$0.00(0.00-0.50)$} & 0.001 \\
\hline DBC-A Depressive subscale (scale range 0-18) & 1024 & \multicolumn{2}{|c|}{$3(1-6)$} & \multicolumn{2}{|c|}{$1(0-4)$} & $<0.0001$ \\
\hline Mean item score (possible score $0-2$ ) & 1024 & \multicolumn{2}{|c|}{$0.33(0.11-0.67)$} & \multicolumn{2}{|c|}{$0.11(0.00-0.44)$} & $<0.0001$ \\
\hline Proportion of items checked (possible score $0-1$ ) & 1024 & \multicolumn{2}{|c|}{$0.33(0.11-0.56)$} & & .33) & $<0.0001$ \\
\hline Intensity index (possible score $0-1$ ) & 718 & & $.39)$ & & .25) & 0.002 \\
\hline Pictorial Epworth Sleepiness Scale (pESS) & 933 & & & & & $<0.0001$ \\
\hline Pictorial Epworth Sleepiness Scale score $>10$ & 933 & 126 & $38.8 \%$ & 89 & $14.6 \%$ & $<0.0001$ \\
\hline Estimated total sleep time (TST) in $24 \mathrm{~h}(\mathrm{~h})$ & 545 & & & & & 0.10 \\
\hline Estimated TST during night (h) & 988 & & & & & 0.02 \\
\hline Estimated TST during daytime (h) & 834 & & & & & $<0.0001$ \\
\hline Naps in daytime & 812 & 111 & $40.8 \%$ & 123 & $22.8 \%$ & $<0.0001$ \\
\hline Snoring—ever ( $\geq 1$ night/week) & & 366 & $100.0 \%$ & 450 & $67.0 \%$ & - \\
\hline Never & & 0 & $0.0 \%$ & 149 & $22.2 \%$ & \\
\hline Rarely/sometimes (1-2 night/week) & 1038 & 0 & $0.0 \%$ & 396 & $58.9 \%$ & $<0.0001$ \\
\hline Often/frequent ( $\geq 3$ nights/week) & & 366 & $100.0 \%$ & 54 & $8.0 \%$ & \\
\hline Don't know & & 0 & $0.0 \%$ & 73 & $10.9 \%$ & - \\
\hline Witnessed apnoeas—ever ( $\geq 1$ night/week) & & 206 & $56.9 \%$ & 103 & $15.7 \%$ & - \\
\hline Never & & 20 & $5.5 \%$ & 339 & $51.8 \%$ & \\
\hline Rarely/sometimes (1-2 night/week) & 1017 & 82 & $22.7 \%$ & 78 & $11.9 \%$ & $<0.0001$ \\
\hline Often/frequent ( $\geq 3$ nights/week) & & 124 & $34.3 \%$ & 25 & $3.8 \%$ & \\
\hline Don't know & & 136 & $37.6 \%$ & 213 & $32.5 \%$ & - \\
\hline Nocturnal choking episodes_ever ( $\geq 1$ night/week) & & 160 & $44.9 \%$ & 117 & $17.6 \%$ & - \\
\hline Never & & 145 & $40.7 \%$ & 495 & $74.4 \%$ & \\
\hline Rarely/sometimes (1-2 night/week) & 1021 & 111 & $31.2 \%$ & 105 & $15.8 \%$ & $<0.0001$ \\
\hline Often/frequent ( $\geq 3$ nights/week) & & 49 & $13.8 \%$ & 12 & $1.8 \%$ & \\
\hline Don't know & & 51 & $14.3 \%$ & 53 & $8.0 \%$ & - \\
\hline Frequent night awakenings-ever ( $\geq 1$ night/week) & & 292 & $81.1 \%$ & 434 & $65.7 \%$ & - \\
\hline Never & & 36 & $10.0 \%$ & 195 & $29.5 \%$ & \\
\hline Rarely/sometimes (1-2 night/week) & 1021 & 158 & $43.9 \%$ & 316 & $47.8 \%$ & $<0.0001$ \\
\hline Often/frequent ( $\geq 3$ nights/week) & & 134 & $37.2 \%$ & 118 & $17.9 \%$ & \\
\hline Don't know & & 32 & $8.9 \%$ & 32 & $4.8 \%$ & - \\
\hline
\end{tabular}


Table 2. Cont.

\begin{tabular}{|c|c|c|c|c|c|c|}
\hline \multirow{2}{*}{$\begin{array}{c}\text { Characteristics } \\
\text { Unrefreshing sleep—ever ( } \geq 1 \text { night/week })\end{array}$} & \multirow[t]{2}{*}{ Total Responses } & \multicolumn{2}{|c|}{$\begin{array}{c}\text { Probable OSA } \\
n=366(34.3 \%)\end{array}$} & \multicolumn{2}{|c|}{$\begin{array}{c}\text { OSA Not Suspected } \\
n=673(63.1 \%)^{*}\end{array}$} & \multirow{2}{*}{$p$} \\
\hline & & 313 & $86.2 \%$ & 431 & $64.9 \%$ & \\
\hline Never & \multirow{4}{*}{1027} & 18 & $5.0 \%$ & 188 & $28.3 \%$ & \multirow{3}{*}{$<0.0001$} \\
\hline Rarely/sometimes (1-2 night/week) & & 118 & $32.5 \%$ & 299 & $45.0 \%$ & \\
\hline Often/frequent ( $\geq 3$ nights/week) & & 195 & $53.7 \%$ & 132 & $19.9 \%$ & \\
\hline Don't know & & 32 & $8.8 \%$ & 45 & $6.8 \%$ & - \\
\hline Daytime sleepiness—ever ( $\geq 1$ night/week) & \multirow{5}{*}{1029} & 322 & $88.7 \%$ & 448 & $67.3 \%$ & - \\
\hline Never & & 36 & $9.9 \%$ & 205 & $30.8 \%$ & \multirow{3}{*}{$<0.0001$} \\
\hline Rarely/sometimes (1-2 night/week) & & 150 & $41.3 \%$ & 335 & $50.3 \%$ & \\
\hline Often/frequent ( $\geq 3$ nights/week) & & 172 & $47.4 \%$ & 113 & $17.0 \%$ & \\
\hline Don't know & & 5 & $1.4 \%$ & 13 & $2.0 \%$ & - \\
\hline
\end{tabular}

* OSA probability could not be calculated for 28 responders $(2.6 \%)$.

Table 3. Anthropometric characteristics of responders meeting criteria for probable OSA on $\geq 1$ algorithm, with responders on CPAP therapy excluded. Chi-square test used for parametric categorical variables, $t$-test for continuous categorical variables and Mann-Whitney $\mathrm{U}$ test for non-parametric variables. Values presented as mean $\pm \mathrm{SD}$ or $n \%$ unless otherwise stated.

\begin{tabular}{|c|c|c|c|c|c|c|}
\hline Characteristics & Total Responses & \multicolumn{2}{|c|}{$\begin{array}{c}\text { Probable OSA } \\
n=366(34.3 \%) *\end{array}$} & \multicolumn{2}{|c|}{$\begin{array}{c}\text { OSA Not Suspected } \\
n=673(63.1 \%)^{*}\end{array}$} & $p$ \\
\hline Age (years) & 1004 & \multicolumn{2}{|c|}{$26 \pm 8$} & \multicolumn{2}{|c|}{$29 \pm 10$} & $<0.0001$ \\
\hline Gender (males:females) & 1037 & \multicolumn{2}{|c|}{ 206:159 } & \multicolumn{2}{|c|}{$363: 309$} & 0.47 \\
\hline Collar size $(\mathrm{cm})$ & 565 & \multicolumn{2}{|c|}{$41 \pm 4.6$} & \multicolumn{2}{|c|}{$40.1 \pm 4.0$} & 0.02 \\
\hline Body Mass Index $\left(\mathrm{kg} / \mathrm{m}^{2}\right)^{* *}$ & 884 & \multicolumn{2}{|c|}{$30.0 \pm 1.3$} & \multicolumn{2}{|c|}{$27.4 \pm 1.2$} & $<0.0001$ \\
\hline Underweight $\left(<18.5 \mathrm{~kg} / \mathrm{m}^{2}\right)$ & \multirow{6}{*}{721} & 0 & $0.0 \%$ & 6 & $1.3 \%$ & \multirow{6}{*}{$<0.0001$} \\
\hline Normal weight $\left(18.5-24.99 \mathrm{~kg} / \mathrm{m}^{2}\right)$ & & 42 & $17.3 \%$ & 140 & $29.3 \%$ & \\
\hline Pre-obesity $(25.0-29.99$ kg/m²) & & 81 & $33.3 \%$ & 168 & $35.1 \%$ & \\
\hline Obesity class I (30.0-34.99 kg/m²) & & 48 & $19.8 \%$ & 100 & $20.9 \%$ & \\
\hline Obesity class II $(35.0-39.99$ kg/m²) & & 44 & $18.1 \%$ & 42 & $8.8 \%$ & \\
\hline Obesity class III $\left(\geq 40.00 \mathrm{~kg} / \mathrm{m}^{2}\right)$ & & 28 & $11.5 \%$ & 22 & $4.6 \%$ & \\
\hline Any medication & 1039 & 273 & $74.6 \%$ & 473 & $70.3 \%$ & 0.001 \\
\hline Benzodiazepines/Z-drugs & 1039 & 7 & $1.9 \%$ & 6 & $0.9 \%$ & 0.24 \\
\hline Opiates & 1039 & 6 & $1.6 \%$ & 6 & $0.9 \%$ & 0.36 \\
\hline Antidepressants & 1039 & 21 & $5.7 \%$ & 33 & $4.9 \%$ & 0.56 \\
\hline Antiepileptics & 1039 & 24 & $6.6 \%$ & 19 & $2.8 \%$ & 0.005 \\
\hline Antihistamines & 1039 & 35 & $9.6 \%$ & 36 & $5.3 \%$ & 0.01 \\
\hline Contraceptives & 1039 & 29 & $7.9 \%$ & 33 & $4.9 \%$ & 0.06 \\
\hline Melatonin & 1039 & 7 & $1.9 \%$ & 9 & $1.3 \%$ & 0.60 \\
\hline Oxygen & 1039 & 4 & $1.1 \%$ & 6 & $0.9 \%$ & 0.75 \\
\hline \multicolumn{7}{|l|}{ Comorbidities: } \\
\hline Asthma & 1039 & 64 & $17.5 \%$ & 68 & $10.1 \%$ & 0.001 \\
\hline Stroke & 1039 & 1 & $0.3 \%$ & 14 & $2.1 \%$ & 0.03 \\
\hline Broken nose & 1039 & 1 & $0.3 \%$ & 6 & $0.9 \%$ & 0.43 \\
\hline Diabetes & 1039 & 7 & $1.9 \%$ & 20 & $3.0 \%$ & 0.42 \\
\hline Heart problems & 1039 & 152 & $41.5 \%$ & 231 & $34.3 \%$ & 0.02 \\
\hline Hay fever & 1039 & 79 & $21.6 \%$ & 111 & $16.5 \%$ & 0.04 \\
\hline Thyroid problems & 1039 & 130 & $35.5 \%$ & 242 & $36.0 \%$ & 0.95 \\
\hline Epilepsy & 1039 & 35 & $9.6 \%$ & 25 & $3.7 \%$ & $<0.0001$ \\
\hline Liver & 1039 & 2 & $0.5 \%$ & 13 & $1.9 \%$ & 0.10 \\
\hline
\end{tabular}


Table 3. Cont.

\begin{tabular}{ccccccc}
\hline Characteristics & Total Responses & \multicolumn{2}{c}{$\begin{array}{c}\text { Probable OSA } \\
n=366(34.3 \%)\end{array}$} & \multicolumn{2}{c}{$\begin{array}{c}\text { OSA Not Suspected } \\
n=673(\mathbf{6 3 . 1} \%)^{*}\end{array}$} \\
\hline Hypertension & 1039 & 10 & $2.7 \%$ & 8 & $1.2 \%$ & 0.08 \\
\hline Nasal surgery & 1039 & 6 & $1.6 \%$ & 8 & $1.2 \%$ & 0.58 \\
\hline Kidney problems & 1039 & 13 & $3.6 \%$ & 9 & $1.3 \%$ & 0.02 \\
\hline Gluten intolerance & 1039 & 20 & $5.5 \%$ & 39 & $5.8 \%$ & 0.89 \\
\hline Any adenoid and/or tonsil surgery & 1039 & 105 & $28.7 \%$ & 135 & $20.1 \%$ & 0.002 \\
\hline
\end{tabular}

* OSA probability could not be calculated for 28 responders (2.6\%); ** WHO BMI category calculated for participants aged $\geq 20$ years only.

Table 4. Self-reported sleep and behaviour characteristics of valid questionnaire responders, with responders on CPAP therapy excluded. Chi-square test used for parametric categorical variables, $t$-test for continuous categorical variables and Mann-Whitney U test for non-parametric variables. Values presented as mean $\pm \mathrm{SD}$, median (IQR) or $n \%$ unless otherwise stated.

\begin{tabular}{|c|c|c|c|c|c|c|c|c|}
\hline Sleep and Behaviour Characteristics & $\begin{array}{c}\text { Total } \\
\text { Responses }\end{array}$ & \multicolumn{2}{|c|}{$\begin{array}{c}\text { All Responders } \\
n=1067\end{array}$} & \multicolumn{2}{|c|}{$\begin{array}{c}\text { Male } \\
n=585(54.8 \%) *\end{array}$} & \multicolumn{2}{|c|}{$\begin{array}{c}\text { Female } \\
n=480(45.0 \%) *\end{array}$} & $p^{* *}$ \\
\hline \multicolumn{9}{|c|}{ Developmental Behaviour Checklist for Adults (DBC-A): } \\
\hline Disruptive behaviour subscale (scale range $0-34$ ) & 1050 & \multicolumn{2}{|c|}{$5(2$ to 10$)$} & \multicolumn{2}{|c|}{$4(2$ to 9$)$} & \multicolumn{2}{|c|}{$6(2$ to 11$)$} & 0.003 \\
\hline Mean item score (possible score $0-2$ ) & 1050 & \multicolumn{2}{|c|}{$0.29(0.12$ to 0.59$)$} & \multicolumn{2}{|c|}{$0.27(0.12$ to 0.55$)$} & \multicolumn{2}{|c|}{0.35 (0.16 to 0.65$)$} & 0.002 \\
\hline Proportion of items checked (possible score $0-1$ ) & 1050 & \multicolumn{2}{|c|}{$0.29(0.12$ to 0.53$)$} & \multicolumn{2}{|c|}{$0.24(0.12$ to 0.47$)$} & \multicolumn{2}{|c|}{$0.29(0.12$ to 0.53$)$} & 0.001 \\
\hline Intensity index (possible score $0-1$ ) & 920 & \multicolumn{2}{|c|}{0.00 (0.00 to 0.27$)$} & \multicolumn{2}{|c|}{$0.00(0.00$ to 0.29$)$} & \multicolumn{2}{|c|}{$0.00(0.00$ to 0.27$)$} & 0.55 \\
\hline Anxiety/Antisocial subscale (scale range -2-14) & 1035 & \multicolumn{2}{|c|}{$0(0$ to 1$)$} & \multicolumn{2}{|c|}{$0(0$ to 1$)$} & \multicolumn{2}{|c|}{$0(-1$ to 1$)$} & 0.96 \\
\hline Mean item score (possible score $0-2$ ) & 1035 & \multicolumn{2}{|c|}{$0.22(0.00$ to 0.33$)$} & \multicolumn{2}{|c|}{$0.22(0.00$ to 0.33$)$} & \multicolumn{2}{|c|}{$0.22(0.11$ to 0.33$)$} & 0.66 \\
\hline Proportion of items checked (possible score $0-1$ ) & 1035 & \multicolumn{2}{|c|}{0.11 (0.00 to 0.33$)$} & \multicolumn{2}{|c|}{$0.11(0.00$ to 0.33$)$} & \multicolumn{2}{|c|}{$0.11(0.11$ to 0.33$)$} & 0.64 \\
\hline Intensity index (possible score $0-1$ ) & 783 & \multicolumn{2}{|c|}{$0.00(0.00$ to 0.50$)$} & \multicolumn{2}{|c|}{$0.00(0.00$ to 0.50$)$} & 0.00( & to 0.50$)$ & 0.91 \\
\hline Depressive subscale (scale range $0-18$ ) & 1050 & & & & & & 5) & 0.17 \\
\hline Mean item score (possible score $0-2$ ) & 1050 & 0.22 & $0.56)$ & 0.22 & $0.56)$ & 0.22( & to 0.56$)$ & 0.04 \\
\hline Proportion of items checked (possible score $0-1$ ) & 1050 & 0.22 & $0.44)$ & 0.22 & $0.44)$ & 0.22( & to 0.44$)$ & 0.03 \\
\hline Intensity index (possible score $0-1$ ) & 735 & 0.00 & $0.33)$ & 0.00 & $0.33)$ & 0.00 & to 0.33$)$ & 0.99 \\
\hline Pictorial Epworth Sleepiness Scale (pESS) & 954 & & & & & & & 0.02 \\
\hline Pictorial Epworth Sleepiness Scale score $>10$ & 954 & 215 & $22.5 \%$ & 124 & $23.8 \%$ & 90 & $20.9 \%$ & 0.31 \\
\hline Estimated total sleep time (TST) in $24 \mathrm{~h}(\mathrm{~h})$ & 559 & & & & & & 1.3 & 0.004 \\
\hline Estimated TST during night $(\mathrm{h})$ & 1011 & & & & & & 1.2 & $<0.0001$ \\
\hline Estimated TST during daytime (h) & 834 & & & & & & $0.5)$ & 0.96 \\
\hline Naps in daytime & 834 & 235 & $28.2 \%$ & 125 & $27.9 \%$ & 109 & $28.3 \%$ & 0.94 \\
\hline Snoring_ever ( $\geq 1$ night/week) & & 830 & $78.9 \%$ & 462 & $80.1 \%$ & 366 & $77.4 \%$ & - \\
\hline Never & & 149 & $14.2 \%$ & 75 & $13.0 \%$ & 74 & $15.6 \%$ & \\
\hline Rarely/sometimes (1-2 night/week) & 1052 & 396 & $37.6 \%$ & 224 & $38.8 \%$ & 171 & $36.2 \%$ & 0.41 \\
\hline Often/frequent ( $\geq 3$ nights/week) & & 434 & $41.3 \%$ & 238 & $41.2 \%$ & 195 & $41.2 \%$ & \\
\hline Don't know & & 73 & $6.9 \%$ & 40 & $6.9 \%$ & 33 & $7.0 \%$ & - \\
\hline Witnessed apnoeas—ever ( $\geq 1$ night/week) & & 309 & $30.0 \%$ & 175 & $30.8 \%$ & 133 & $29.0 \%$ & - \\
\hline Never & & 370 & $36.0 \%$ & 200 & $35.2 \%$ & 169 & $36.8 \%$ & \\
\hline Rarely/sometimes (1-2 night/week) & 1029 & 160 & $15.5 \%$ & 83 & $14.6 \%$ & 76 & $16.6 \%$ & 0.19 \\
\hline Often/frequent ( $\geq 3$ nights/week) & & 149 & $14.5 \%$ & 92 & $16.2 \%$ & 57 & $12.4 \%$ & \\
\hline Don't know & & 350 & $34.0 \%$ & 193 & $34.0 \%$ & 157 & $34.2 \%$ & - \\
\hline Nocturnal choking episodes-ever ( $\geq 1$ night/week) & & 282 & $27.1 \%$ & 142 & $24.9 \%$ & 140 & $29.9 \%$ & - \\
\hline Never & & 656 & $63.0 \%$ & 362 & $63.4 \%$ & 292 & $62.3 \%$ & \\
\hline Rarely/sometimes (1-2 night/week) & 1042 & 221 & $21.2 \%$ & 111 & $19.4 \%$ & 110 & $23.5 \%$ & 0.37 \\
\hline Often/frequent ( $\geq 3$ nights/week) & & 61 & $5.9 \%$ & 31 & $5.4 \%$ & 30 & $6.4 \%$ & \\
\hline Don't know & & 104 & $10.0 \%$ & 67 & $11.7 \%$ & 37 & $7.9 \%$ & - \\
\hline
\end{tabular}


Table 4. Cont.

\begin{tabular}{|c|c|c|c|c|c|c|c|c|}
\hline \multirow{2}{*}{$\begin{array}{l}\text { Sleep and Behaviour Characteristics } \\
\text { Frequent night awakenings—ever ( } \geq 1 \text { night/week) }\end{array}$} & \multirow[t]{2}{*}{$\begin{array}{c}\text { Total } \\
\text { Responses }\end{array}$} & \multicolumn{2}{|c|}{$\begin{array}{c}\text { All Responders } \\
n=1067\end{array}$} & \multicolumn{2}{|c|}{$\begin{array}{c}\text { Male } \\
n=585(54.8 \%)\end{array}$} & \multicolumn{2}{|c|}{$\begin{array}{c}\text { Female } \\
n=480(45.0 \%)\end{array}$} & \multirow{2}{*}{$p^{* *}$} \\
\hline & & 743 & $71.2 \%$ & 393 & $68.7 \%$ & 349 & $74.3 \%$ & \\
\hline Never & \multirow{4}{*}{1044} & 237 & $22.7 \%$ & 235 & $41.1 \%$ & 101 & $21.5 \%$ & \multirow{3}{*}{0.36} \\
\hline Rarely/sometimes (1-2 night/week) & & 484 & $46.4 \%$ & 262 & $45.8 \%$ & 222 & $47.2 \%$ & \\
\hline Often/frequent ( $\geq 3$ nights/week) & & 259 & $24.8 \%$ & 131 & $22.9 \%$ & 127 & $27.0 \%$ & \\
\hline Don't know & & 64 & $6.1 \%$ & 44 & $7.7 \%$ & 20 & $4.3 \%$ & - \\
\hline Unrefreshing sleep—ever ( $\geq 1$ night/week) & \multirow{5}{*}{1047} & 760 & $72.6 \%$ & 413 & $72.3 \%$ & 470 & $73.0 \%$ & - \\
\hline Never & & 210 & $20.1 \%$ & 114 & $20.0 \%$ & 95 & $20.0 \%$ & \multirow{3}{*}{0.90} \\
\hline Rarely/sometimes (1-2 night/week) & & 428 & $40.9 \%$ & 236 & $41.3 \%$ & 192 & $40.5 \%$ & \\
\hline Often/frequent ( $\geq 3$ nights/week) & & 332 & $31.7 \%$ & 177 & $31.0 \%$ & 154 & $32.5 \%$ & \\
\hline Don't know & & 77 & $7.4 \%$ & 44 & $7.7 \%$ & 33 & $7.0 \%$ & - \\
\hline Daytime sleepiness—ever ( $\geq 1$ night/week) & \multirow{5}{*}{1050} & 787 & $75.0 \%$ & 443 & $93.1 \%$ & 342 & $72.5 \%$ & - \\
\hline Never & & 244 & $23.2 \%$ & 121 & $25.4 \%$ & 123 & $26.1 \%$ & \multirow{3}{*}{0.11} \\
\hline Rarely/sometimes (1-2 night/week) & & 500 & $47.6 \%$ & 276 & $58.0 \%$ & 224 & $47.5 \%$ & \\
\hline Often/frequent ( $\geq 3$ nights/week) & & 287 & $27.3 \%$ & 167 & $35.1 \%$ & 118 & $25.0 \%$ & \\
\hline Don't know & & 19 & $1.8 \%$ & 12 & $2.5 \%$ & 7 & $1.5 \%$ & - \\
\hline \multicolumn{9}{|c|}{ Obstructive sleep apnoea (OSA) status: } \\
\hline Prior diagnosis of OSA & 1067 & 44 & $4.1 \%$ & 29 & $5.0 \%$ & 15 & $3.1 \%$ & 0.16 \\
\hline Probable OSA using definition 1 & 1038 & 350 & $33.7 \%$ & 199 & $34.9 \%$ & 150 & $32.2 \%$ & 0.39 \\
\hline Probable OSA using definition 2 & 1042 & 356 & $34.2 \%$ & 200 & $35.1 \%$ & 155 & $33.0 \%$ & 0.51 \\
\hline Probable OSA using definition 3 & 1038 & 352 & $33.9 \%$ & 200 & $35.1 \%$ & 151 & $32.4 \%$ & 0.39 \\
\hline Probable OSA on $\geq 1$ definition & 1039 & 366 & $35.2 \%$ & 206 & $36.2 \%$ & 159 & $34.0 \%$ & 0.47 \\
\hline
\end{tabular}

* Gender of 2 responders unknown; ${ }^{* *}$ Difference between males and females.

The mean self-reported total sleep time was $9.1 \pm 1.3 \mathrm{~h}$ in $24 \mathrm{~h}$, with $8.6 \pm 1.2 \mathrm{~h}$ of nocturnal sleep. Most participants (72\%) did not take daytime naps. Nocturnal total sleep time was significantly higher in females $(<0.0001)$.

\subsection{Behavioural and Emotional Disturbances}

As hypothesised, OSAHS was a strong marker for diurnal behavioural disturbances; individuals with probable OSAHS scored significantly higher on all three DBC-A domains (Table 2). Independent determinants of sleepiness and behavioural and emotional disturbances are summarised in Tables 5 and 6, and include use of category $\mathrm{N}$ medications, unrefreshing sleep and frequent nocturnal awakenings.

Table 5. Determinants of sleepiness and probable obstructive sleep apnoea/hypopnoea syndrome as assessed by binary logistic regression $(\mathrm{OR}) *$ or generalised linear modelling $(\beta)^{* *}$ as appropriate for categorical and continuous variables, respectively. DBC-A Disruptive subscale analysed separately for males and females due to significant difference in scores at baseline.

\begin{tabular}{|c|c|c|c|c|c|c|}
\hline Variable & Total Included & Determinants Remaining in Model & Estimate & 95\% CI Lower & 95\% CI Upper & $p$ \\
\hline \multirow{7}{*}{$\begin{array}{l}\text { Pictorial Epworth } \\
\text { Sleepiness Scale ** }\end{array}$} & & Age & 0.0 & 0.0 & 0.1 & 0.07 \\
\hline & & BMI & 0.1 & 0.0 & 0.2 & 0.004 \\
\hline & & Hay fever & 1.3 & 0.2 & 2.4 & 0.02 \\
\hline & & Category G medication & 2.2 & 0.5 & 3.9 & 0.01 \\
\hline & & Category N medication & 2.0 & 0.8 & 3.1 & 0.001 \\
\hline & & $\begin{array}{c}\text { Witnessed } \\
\text { apnoeas_rarely/sometimes } \\
(1-2 \text { nights/week })\end{array}$ & 1.8 & 0.8 & 2.8 & 0.001 \\
\hline & & $\begin{array}{l}\text { Witnessed apnoeas—often/frequently } \\
\text { ( } \geq 3 \text { nights/week) }\end{array}$ & 5.5 & 4.4 & 6.6 & $<0.0001$ \\
\hline
\end{tabular}


Table 5. Cont.

\begin{tabular}{|c|c|c|c|c|c|c|}
\hline Variable & Total Included & Determinants Remaining in Model & Estimate & 95\% CI Lower & 95\% CI Upper & $p$ \\
\hline \multirow{5}{*}{$\begin{array}{c}\text { Excessive daytime } \\
\text { sleepiness }(p E S S>10)\end{array}$} & & Age & 1.0 & 1.0 & 1.1 & 0.14 \\
\hline & & BMI & 1.0 & 1.0 & 1.1 & 0.08 \\
\hline & & Category $\mathrm{N}$ medication & 0.4 & 0.2 & 0.7 & 0.001 \\
\hline & & $\begin{array}{c}\text { Witnessed } \\
\text { apnoeas_rarely/sometimes } \\
\text { (1-2 nights/week) }\end{array}$ & 0.1 & 0.1 & 0.2 & $<0.0001$ \\
\hline & & $\begin{array}{l}\text { Witnessed apnoeas—often/frequently } \\
\text { ( } \geq 3 \text { nights/week) }\end{array}$ & 0.3 & 0.2 & 0.6 & $<0.0001$ \\
\hline \multirow{4}{*}{$\begin{array}{c}\text { Probable obstructive sleep } \\
\text { apnoea/hypopnoea } \\
\text { syndrome }\end{array}$} & & Age & 1.0 & 0.9 & 1.0 & $<0.0001$ \\
\hline & & BMI & 1.1 & 1.1 & 1.1 & $<0.0001$ \\
\hline & & Epilepsy & 0.2 & 0.1 & 0.5 & $<0.0001$ \\
\hline & & Category R medication & 0.6 & 0.4 & 0.9 & 0.01 \\
\hline
\end{tabular}

Table 6. Determinants of behavioural and emotional disturbances as assessed by generalised linear modelling ( $\beta$ ) for continuous variables. DBC-A Disruptive subscale analysed separately for males and females due to significant difference in scores at baseline.

\begin{tabular}{|c|c|c|c|c|c|c|}
\hline Variable & Total Included & Determinants Remaining in Model & Estimate $(\beta)$ & 95\% CI Lower & 95\% CI Upper & $p$ \\
\hline \multirow{11}{*}{$\begin{array}{c}\text { DBC-A } \\
\text { Disruptive-Male }\end{array}$} & & Category N medication & 3.0 & 1.2 & 4.8 & 0.001 \\
\hline & & Snoring—rarely/sometimes (1-2 nights/week) & -1.8 & -3.4 & -0.1 & 0.04 \\
\hline & & Snoring—often/frequently ( $\geq 3$ nights/week) & -1.4 & -3.3 & 0.6 & 0.16 \\
\hline & & $\begin{array}{l}\text { Witnessed apnoeas-rarely/sometimes } \\
\text { (1-2 nights/week) }\end{array}$ & 2.0 & 0.3 & 3.7 & 0.02 \\
\hline & & $\begin{array}{l}\text { Witnessed apnoeas—often/frequently } \\
\text { ( } \geq 3 \text { nights/week) }\end{array}$ & 1.5 & -0.5 & 3.5 & 0.14 \\
\hline & & $\begin{array}{l}\text { Nocturnal choking_rarely/sometimes } \\
\text { (1-2 nights/week) }\end{array}$ & 1.4 & -2.1 & 3.0 & 0.09 \\
\hline & & $\begin{array}{l}\text { Nocturnal choking_often/frequently } \\
(\geq 3 \text { nights/week) }\end{array}$ & -0.4 & -3.2 & 2.4 & 0.77 \\
\hline & & $\begin{array}{l}\text { Frequent awakenings-rarely/sometimes } \\
\text { (1-2 nights/week) }\end{array}$ & 1.8 & 0.3 & 3.3 & 0.02 \\
\hline & & $\begin{array}{l}\text { Frequent awakenings_often/frequently } \\
(\geq 3 \text { nights/week })\end{array}$ & 3.7 & 1.7 & 5.7 & $<0.0001$ \\
\hline & & $\begin{array}{l}\text { Unrefreshing sleep-rarely/sometimes } \\
\text { (1-2 nights/week) }\end{array}$ & -0.1 & -1.7 & 1.4 & 0.85 \\
\hline & & $\begin{array}{c}\text { Unrefreshing sleep—often/frequently } \\
\text { ( } \geq 3 \text { nights/week) }\end{array}$ & 2.1 & -0.2 & 4.3 & 0.07 \\
\hline \multirow{11}{*}{$\begin{array}{c}\text { DBC-A } \\
\text { Disruptive-Female }\end{array}$} & & Category N medication & 4.1 & 2.6 & 5.6 & $<0.0001$ \\
\hline & & Snoring_rarely/sometimes (1-2 nights/week) & 0.6 & -1.0 & 2.2 & 0.47 \\
\hline & & Snoring—often/frequently ( $\geq 3$ nights/week) & 0.5 & -1.2 & 2.3 & 0.54 \\
\hline & & $\begin{array}{l}\text { Witnessed apnoeas-rarely/sometimes } \\
\text { (1-2 nights/week) }\end{array}$ & -1.6 & -3.1 & -0.1 & 0.04 \\
\hline & & $\begin{array}{l}\text { Witnessed apnoeas—often/frequently } \\
\text { ( } \geq 3 \text { nights/week) }\end{array}$ & -0.8 & -3.2 & 1.5 & 0.48 \\
\hline & & $\begin{array}{l}\text { Nocturnal choking-rarely/sometimes } \\
\text { (1-2 nights/week) }\end{array}$ & 2.6 & 1.1 & 4.1 & 0.00 \\
\hline & & $\begin{array}{c}\text { Nocturnal choking_often/frequently } \\
(\geq 3 \text { nights/week) }\end{array}$ & 3.9 & 1.0 & 6.7 & 0.01 \\
\hline & & $\begin{array}{l}\text { Frequent awakenings-rarely/sometimes } \\
\text { (1-2 nights/week) }\end{array}$ & 1.4 & -0.1 & 2.9 & 0.08 \\
\hline & & $\begin{array}{l}\text { Frequent awakenings_often/frequently } \\
\text { ( } \geq 3 \text { nights/week) }\end{array}$ & -0.2 & -2.1 & 1.7 & 0.85 \\
\hline & & $\begin{array}{l}\text { Unrefreshing sleep-rarely/sometimes } \\
\text { (1-2 nights/week) }\end{array}$ & 1.5 & 0.0 & 3.0 & 0.06 \\
\hline & & $\begin{array}{l}\text { Unrefreshing sleep—often/frequently } \\
\text { ( } \geq 3 \text { nights/week) }\end{array}$ & 6.2 & 4.3 & 8.1 & $<0.0001$ \\
\hline
\end{tabular}


Table 6. Cont.

\begin{tabular}{|c|c|c|c|c|c|c|}
\hline Variable & Total Included & Determinants Remaining in Model & Estimate $(\beta)$ & 95\% CI Lower & 95\% CI Upper & $p$ \\
\hline \multirow{14}{*}{$\begin{array}{c}\text { DBC-A } \\
\text { Anxiety/Antisocial }\end{array}$} & & Category $\mathrm{R}$ medication & 0.3 & 0.0 & 0.6 & 0.09 \\
\hline & & Category $\mathrm{N}$ medication & 0.1 & -0.2 & 0.4 & 0.56 \\
\hline & & Snoring_rarely/sometimes (1-2 nights/week) & 0.0 & -0.4 & 0.3 & 0.82 \\
\hline & & Snoring—often/frequently ( $\geq 3$ nights/week) & -0.3 & -0.7 & 0.1 & 0.13 \\
\hline & & $\begin{array}{l}\text { Witnessed apnoeas_rarely/sometimes } \\
\qquad(1-2 \text { nights/week) }\end{array}$ & 0.2 & -0.2 & 0.5 & 0.32 \\
\hline & & $\begin{array}{l}\text { Witnessed apnoeas—often/frequently } \\
\text { ( } \geq 3 \text { nights/week) }\end{array}$ & -0.1 & -0.5 & 0.3 & 0.67 \\
\hline & & $\begin{array}{l}\text { Nocturnal choking-rarely/sometimes } \\
\text { (1-2 nights/week) }\end{array}$ & 0.3 & 0.0 & 0.7 & 0.03 \\
\hline & & $\begin{array}{l}\text { Nocturnal choking-often/frequently } \\
\text { ( } \geq 3 \text { nights/week) }\end{array}$ & 0.5 & -0.1 & 1.1 & 0.11 \\
\hline & & $\begin{array}{c}\text { Frequent awakenings-rarely/sometimes } \\
\qquad(1-2 \text { nights/week) }\end{array}$ & 0.1 & -0.2 & 0.4 & 0.55 \\
\hline & & $\begin{array}{l}\text { Frequent awakenings—often/frequently } \\
\qquad(\geq 3 \text { nights/week })\end{array}$ & 0.0 & -0.4 & 0.4 & 0.87 \\
\hline & & $\begin{array}{l}\text { Unrefreshing sleep—rarely/sometimes } \\
\text { (1-2 nights/week) }\end{array}$ & 0.0 & -0.3 & 0.3 & 1.00 \\
\hline & & $\begin{array}{l}\text { Unrefreshing sleep-often/frequently } \\
\text { ( } \geq 3 \text { nights/week) }\end{array}$ & 0.5 & 0.1 & 1.0 & 0.03 \\
\hline & & $\begin{array}{l}\text { Daytime sleepiness-rarely/sometimes } \\
\text { (1-2 nights/week) }\end{array}$ & 0.2 & -0.1 & 0.5 & 0.20 \\
\hline & & $\begin{array}{l}\text { Daytime sleepiness—often/frequently } \\
\text { ( } \geq 3 \text { nights/week) }\end{array}$ & 0.5 & 0.1 & 0.9 & 0.01 \\
\hline \multirow{14}{*}{ DBC-A Depressive } & & Category $\mathrm{R}$ medication & 0.0 & -0.7 & 0.7 & 1.00 \\
\hline & & Category $\mathrm{N}$ medication & 2.3 & 1.6 & 3.0 & $<0.0001$ \\
\hline & & Snoring—rarely/sometimes (1-2 nights/week) & -0.2 & -0.9 & 0.5 & 0.50 \\
\hline & & Snoring—often/frequently ( $\geq 3$ nights/week) & -0.2 & -1.0 & 0.5 & 0.55 \\
\hline & & $\begin{array}{l}\text { Witnessed apnoeas_rarely/sometimes } \\
\qquad(1-2 \text { nights/week) }\end{array}$ & 0.4 & -0.3 & 1.1 & 0.22 \\
\hline & & $\begin{array}{l}\text { Witnessed apnoeas—often/frequently } \\
\text { ( } \geq 3 \text { nights/week) }\end{array}$ & 0.9 & 0.0 & 1.8 & 0.06 \\
\hline & & $\begin{array}{l}\text { Nocturnal choking_rarely/sometimes } \\
\text { (1-2 nights/week) }\end{array}$ & 0.5 & -0.2 & 1.1 & 0.16 \\
\hline & & $\begin{array}{l}\text { Nocturnal choking_often/frequently } \\
\text { ( } \geq 3 \text { nights/week) }\end{array}$ & 0.5 & -0.7 & 1.6 & 0.46 \\
\hline & & $\begin{array}{l}\text { Frequent awakenings-rarely/sometimes } \\
\qquad(1-2 \text { nights/week) }\end{array}$ & 0.5 & -0.2 & 1.1 & 0.15 \\
\hline & & $\begin{array}{l}\text { Frequent awakenings_often/frequently } \\
\qquad(\geq 3 \text { nights/week })\end{array}$ & 0.3 & -0.6 & 1.1 & 0.52 \\
\hline & & $\begin{array}{l}\text { Unrefreshing sleep—rarely/sometimes } \\
\text { (1-2 nights/week) }\end{array}$ & 0.5 & -0.2 & 1.1 & 0.19 \\
\hline & & $\begin{array}{c}\text { Unrefreshing sleep—often/frequently } \\
\text { ( } \geq 3 \text { nights/week) }\end{array}$ & 2.3 & 1.4 & 3.3 & $<0.0001$ \\
\hline & & $\begin{array}{c}\text { Daytime sleepiness-rarely/sometimes } \\
\text { (1-2 nights/week) }\end{array}$ & -0.1 & -0.7 & 0.5 & 0.67 \\
\hline & & $\begin{array}{c}\text { Daytime sleepiness—often/frequently } \\
(\geq 3 \text { nights/week) }\end{array}$ & 0.5 & -0.3 & 1.3 & 0.23 \\
\hline
\end{tabular}

\subsection{Home Sleep Apnoea Testing}

A summary of questionnaire responders entering the objective prevalence study is shown in Figure 2. Of the 1067 valid responders not currently receiving CPAP therapy, $277(26 \%)$ did not wish to be contacted further. Of the 790 invitations sent, 427 responses were received (54\%), 260 of which declined to participate. Although a reason for declining was not sought, a number of responders annotated their reply slip with a reason; common reasons for declining participation included belief that the individual with Down syndrome would not tolerate the equipment or cope with the study $(17 \%)$, the logistics of the study or general family circumstances making participation problematic $(10 \%)$, other co-existing 
comorbidities or disabilities (7\%), and a general dislike of hospitals/medical intervention $(4 \%)$. Twelve individuals $(5 \%)$ declined to participate because they did not perceive themselves to have a sleep problem. Six individuals reported that they were on CPAP or other sleep treatments and so were ineligible for further inclusion.

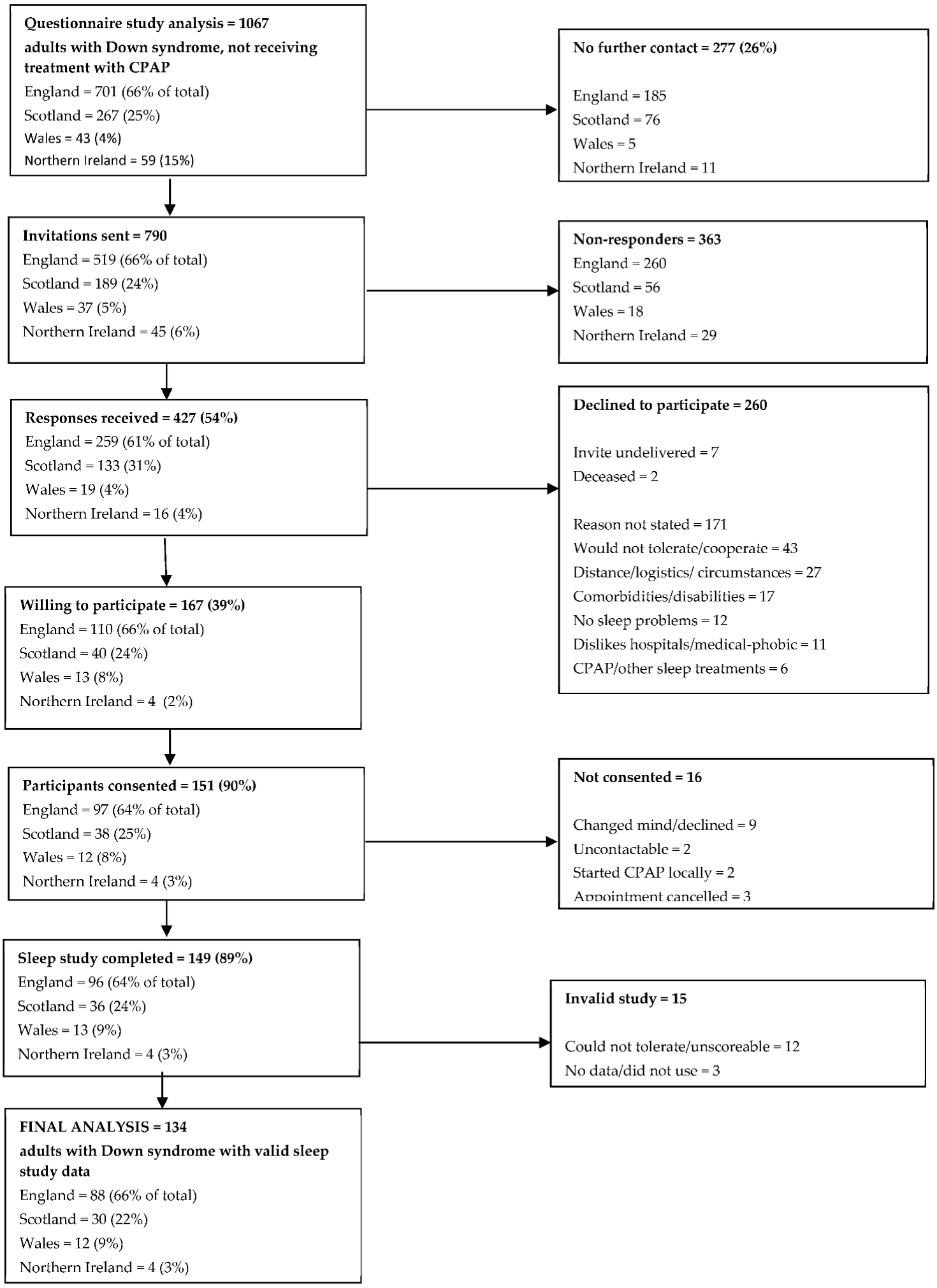

Figure 2. Summary of questionnaire responders entering the objective prevalence study. 
One hundred and sixty-seven individuals were willing to undergo a home sleep study. Of these, 151 (90\%) completed informed consent and were formally recruited into the study. Of those who did not consent, nine changed their mind and declined to participate, two could not be contacted to book an appointment and a further two had commenced CPAP through a local clinical service since returning a reply slip. Three individuals cancelled appointments and were not rebooked.

In total, 149 individuals underwent home sleep studies. Of the recorded studies, 15 studies $(10 \%)$ did not yield valid results, with 12 studies being unscoreable or not tolerated, and 3 studies having no data recorded or not been used and were not repeated. Therefore, the final analysis is based on 134 adults with Down syndrome with valid home sleep study data.

The total scored recording time averaged $488.4 \pm 115.1 \mathrm{~min}(8.1 \pm 2.6 \mathrm{~h})$, similar to the estimated TST at night reported subjectively via questionnaire $(8.4 \pm 1.2 \mathrm{~h})$, and did not differ significantly between genders. An AHI $\geq 15$ or an ODI $\geq 10 / \mathrm{h}$ is considered diagnostic of OSA; $62 \%$ met the criteria for AHI and $41 \%$ the criteria for ODI. The probable OSAHS algorithms [14] were adapted, to allow direct comparison of the subjective diagnosis of probable OSAHS with the objective diagnosis of OSAHS by substituting snoring $\geq 3$ nights/week and witnessed apnoeas with $\mathrm{AHI} \geq 15$ :

1. $\mathrm{AHI} \geq 15$ plus $\mathrm{pESS}>10 / 24$

2. $\quad \mathrm{AHI} \geq 15$ plus unrefreshing sleep $\geq 3$ nights per week.

3. $\mathrm{AHI} \geq 15$ plus daytime sleepiness $\geq 3$ nights per week.

Using these modified algorithms, $42 \%$ of participants demonstrated OSAHS on $\geq 1$ definition (versus $56 \%$ of the same group who met the criteria for probable OSAHS).

Subjective and objective measures of OSAHS were compared. Sensitivity of the probable OSAHS algorithms (ability of the algorithms to correctly identify individuals with objectively diagnosed OSAHS) was 79.2\%, with a specificity (ability of the algorithms to correctly identify individuals who do not have OSAHS) of $58.5 \%$. The positive predictive value (PPV; the likelihood of an individual to have OSAHS in the event of meeting the criteria for probable OSAHS via the algorithms) was $58.5 \%$ and the negative predictive value (NPV; the likelihood of an individual to be OSAHS negative in the event of obtaining a negative result on the algorithms) was 79.2\%. The likelihood ratio (how much more likely it is that an individual who is positive for probable OSAHS via the algorithms will have an objective diagnosis of OSAHS) was 1.9.

\section{Discussion}

This is the first large-scale prevalence study of OSAHS in adults with Down syndrome. Based on self-reported symptoms [14], the prevalence of OSAHS was approximately 37\%, of whom only $38 / 404(9 \%)$ had received a prior diagnosis. Using objective methods, a similar prevalence of $42 \%$ was observed

This mean prevalence of $40 \%$ is modest in comparison with the $>80 \%$ quoted in previous prevalence studies for the Down syndrome population, though these studies were limited by being extremely small scale $[7,8]$. The $40 \%$ prevalence is substantially higher than the $2-4 \%$ prevalence reported in the general population [4]. Clearly, OSAHS is being markedly overlooked in the clinical care of adults with Down syndrome, with only 3\% of this cohort already diagnosed with and receiving treatment for OSAHS. This may be related to diagnostic overshadowing, leading to potentially inappropriate treatments for behavioural and emotional disturbances which may result directly from poor sleep but are not being recognised as such.

\subsection{Acceptability of Home Sleep Apnoea Testing}

Home sleep apnoea testing with the Embletta ${ }^{\circledR}$ Gold ${ }^{\mathrm{TM}}$ cardiorespiratory polygraphy device was well-tolerated by the participants, with a success rate of $90 \%$ over two nights' recording. This is similar to previously published data on the use of home sleep apnoea testing equipment in the general population [18]. Home sleep apnoea testing is common- 
place clinically in the UK and Europe, offering logistical as well as financial benefits over inpatient polysomnography [15]. People with Down syndrome may be medical-phobic or find in-patient visits distressing, so adequate testing in the home environment to make a diagnosis of OSAHS as accessible as possible is vital.

\subsection{Use of the $\mathrm{PESS}$ in Down Syndrome}

A discrepancy was noted between self-reporting of daytime sleepiness per se and the percentage of those reporting elevated pESS scores. Although the pESS appears to be a useful measure in this population, overall scores may have been reduced due to the unsuitability of some of the questions. The question regarding sitting down and reading may not be appropriate given the diminished literacy in this population, and may be problematic in those with visual impairment. With regard to the question related to afternoon napping, $69 \%$ of responders indicated via the pESS that they were likely to nap in the afternoon should circumstances permit, despite only $28 \%$ of respondents reporting daytime napping. This may reflect a lack of opportunity to nap (due to employment, education or other daytime commitments) rather than absence of sleepiness. Modification of the pESS to improve its utility in this specific population may be appropriate.

\subsection{Self-Reporting of Nocturnal Symptoms}

A large percentage of responders did not know whether or not they had apnoeas, which may have resulted in underestimation of the prevalence of this symptom. This may reflect the relative complexity of the description (people are generally more familiar with snoring than "pauses in breathing"), but may also relate to the availability of a second individual to witness the apnoeas; many people with Down syndrome live in supported accommodation without a live-in caregiver or family member and, whereas snoring may be heard outside the bedroom, apnoeas are not. This may also explain the relatively young mean age of respondents, who may still reside at home. However, this is speculative, given that information on living arrangements was not recorded in this study.

All responders with probable OSAHS reported snoring $\geq 3$ nights per week, as this variable was common to all three algorithms. Sleep apnoea without snoring is rare in the literature, though one polysomnographic study in adults with Down syndrome [19] noted snoring in only 7 of 12 participants with studies diagnostic of sleep apnoea. The algorithms used in the current study may be under-estimating the prevalence of OSAHS by excluding those who snored $<3$ nights per week.

\subsection{Behavioural and Emotional Disturbances}

Generally, scores on the subscales of the DBC-A were low. A floor effect was noted on the Anxiety/Antisocial subscale, with mean scores of $0 / 14$ in both males and females, regardless of sleep symptoms. There may be an element of selection bias, with families of individuals with more severe behavioural and emotional problems less likely to respond. However, probable OSAHS was associated with significant increases in raw and mean scores as well as the breadth and intensity of problem behaviours across all three subscales, supporting the hypothesis of OSAHS impacting negatively on behaviour and emotion in adults with Down syndrome. This may lead to inappropriate prescribing of psychotropic drugs. Given their side effects, and the difficulties that people with Down syndrome may have in reporting side effects due to cognitive and communication impairments, this may cause further unnecessary suffering.

Cognitive and behavioural deficits in adults and children with untreated OSAHS are well documented in the general adult population, as is reversal of these deficits with treatment [4]. Since adults with Down syndrome already exhibit cognitive impairment, untreated sleep-disordered breathing may present a "double-hit" on cognition in these individuals. It is possible that untreated sleep-disordered breathing may contribute to the acceleration of the cognitive decline seen in early onset dementia, which is common in adults with Down syndrome [3]; a recent review by Fernandez and Edgin [7] suggests that 
sleep disruption might lead to both earlier onset of dementia and more rapid deterioration. However, to date, no published studies have investigated the effect of sleep-disordered breathing on cognitive function in adults with Down syndrome, nor the effect of CPAP therapy in this group. A recent paper by Cody et al. [20] has suggested that disrupted sleep in adults with Down syndrome was more likely to be associated with impaired cognitive functioning and higher striatal beta amyloid deposition.

\subsection{Adenotonsillectomy}

Adenotonsillectomy is the first-line treatment for OSAHS in the majority of typically developing children, curative in $75-100 \%$, although previous studies report less favourable results in children with Down syndrome [21]. In our study, previous surgery did not result in a lower rate of excessive daytime sleepiness, and those who had previously had adenoids and/or tonsils removed were more likely to exhibit witnessed apnoeas and trended towards reporting more Disruptive and Depressive behaviour. Whilst surgery may result in a partial or initial improvement, results are not sustained into adulthood in the Down syndrome population. This is supported by a previous study in a population of adolescents and young adults (age 14-30 years) with Down syndrome [22].

\subsection{Limitations of the Study}

Inherent non-responder bias in questionnaire studies is well-documented, and difficult to avoid [23]; however, we believe that the questionnaire was designed in such a way as to minimise this. An element of selection bias may be evident, with those individuals and families with concerns about sleep more likely to respond.

There was some regional variation in method. An England-based charity which sent out the majority $(3895 ; 74 \%$ ) of the questionnaires in England, Wales and Northern Ireland declined to send out a second questionnaire to individuals who did not respond initially. A repeat mailout was conducted by all other services involved in the study. This may have contributed to the reduced response rate in these countries.

Although the questionnaire was designed to be completed by the individual with Down syndrome, it is likely that a large proportion of the questionnaires were completed by a proxy on the participant's behalf. A "proxy effect" has been reported in the literature [24]. However, since this more often results in under-reporting of characteristics, it is likely that our estimates of prevalence, sleepiness and behaviour are conservative. The approach we utilized in determining a positive or negative diagnosis of OSAHS appeared to be reasonable, but diagnosis by questionnaire and self-report is always fraught with bias, and objective measurement of sleep disordered breathing is always preferable. In the future, better questionnaire methods may be devised that are more specific to the Down syndrome group.

The majority of participants were identified through patient support groups, and so may not be representative of the Down syndrome population as a whole. Older adults may be under-represented, possibly due to inability to complete the questionnaire on account of comorbidities such as dementia, or absence of a living relative or family member to assist with completion.

One final comment regarding the validity of our results pertains to non-responders. At the time of our study, we did not have the facility to sample non-responders with respect to symptoms of sleep disordered breathing in order to assess whether the prevalence was equally high —a bias which should be controlled for in future studies.

Information on ethnicity was not collected, and our prevalence data may not be transferable to other countries with divergent ethnicity. However, work comparing the prevalence of OSAHS in adults with Down syndrome in Scotland with that in Japan showed similar prevalence of symptoms, despite the ethnic and anthropometric differences between these two populations [25].

This study focussed only on symptoms of OSAHS. However, several symptoms such as excessive daytime somnolence, unrefreshing sleep and frequent night wakening are 
common to other sleep disorders, which cannot be ruled out as co-morbid or alternative causes for these symptoms. Questionnaire assessment of any disorder, including OSAHS, generally results in a lower specificity than sensitivity and we advocate testing all patients where there is any suspicion of sleep-disordered breathing to ensure it is not missed.

Building on this work, objective sleep study data could further quantify the severity of sleep-disordered breathing in adults with Down syndrome. The reliability of home sleep apnoea testing is now validated for the diagnosis of sleep disordered breathing in adults in comparison to polysomnography [15]. However, it should be noted that there is, in general, a difference of about $20 \%$ between home testing and in-lab PSG with respect to the apnoea-hypopnoea index [26]. Although patients with OSAHS and Down syndrome should be offered treatment with continuous positive airway pressure therapy, a strong evidence base for this was lacking at the time our study was conceived of and commenced (2010-2011).

\section{Conclusions}

In conclusion, this first large-scale study of OSAHS prevalence in adults with Down syndrome shows an estimate of 37\%-nearly 9 times higher than in the general adult population. Unfortunately, neither assessment nor treatment of OSAHS in adults with Down syndrome appears to be common clinical practice, as evidenced by only $7 \%$ of the study population having a prior diagnosis of OSA and 3.4\% receiving CPAP treatment, despite the potential benefits for improved cognitive function, health and wellbeing. This study strengthens the evidence for guidelines for the monitoring of OSAHS in Down syndrome adults and the establishment of specialised clinics for adults with Down syndrome and other forms of intellectual disabilities. We argue for improved access to these services and measures to improve awareness of this disorder amongst people with Down syndrome, their families and all professionals involved in their care.

Author Contributions: E.A.H. led the study, collected and analysed data and wrote the initial draft of the MS; L.J.W. led data analysis and contributed to review and editing of the study; S.-A.C. contributed to the methodology, ethical approval, review and editing of the study; R.L.R. conceptualised the project, contributed to the methodology, supervision, review and editing of the study and obtained the funding for the study. All authors have read and agreed to the published version of the manuscript.

Funding: This study was funded by the following sources: Baily Thomas Charitable Fund (Trust/RNA/ AC/TM/2634-5178); Chief Scientist's Office (CZH/4/549); and the Fondation Jérôme Lejeune (July 2010).

Institutional Review Board Statement: The study was approved by the Scotland A Research Ethics Committee (REC no: 11/MRE00/3). Return of a completed questionnaire was considered implicit consent to participate. Individuals undertaking home sleep apnoea testing gave written informed consent.

Informed Consent Statement: Informed consent was obtained from all subjects involved in the study.

Data Availability Statement: The authors are happy to share data utilised in the analyses detailed above on personal request to the first or last author and with the full permission of all authors in this study.

Acknowledgments: The authors wish to thank the individuals and families who participated in the study. We acknowledge the vital roles played by Down's Syndrome Scotland, the Down's Heart Group and the Down's Syndrome Association in identifying participants, mailing questionnaires and supporting the study. John Taffe, Centre for Developmental Psychiatry and Psychology, Monash University, provided invaluable assistance with scoring and interpretation of the DBC-A. We acknowledge the work of medical students and others within the Sleep Research Unit who assisted with questionnaire preparation and data entry.

Conflicts of Interest: The authors declare no conflict of interest. 


\section{References}

1. Loane, M.; Morris, J.; Addor, M.-C.; Arriola, L.; Budd, J.; Doray, B.; Garne, E.; Gatt, M.; Haeusler, M.; Khoshnood, B.; et al. Twenty-year trends in the prevalence of down syndrome and other trisomies in Europe: Impact of Maternal Age and Prenatal Screening. Eur. J. Hum. Genet. 2013, 21, 27-33. [CrossRef]

2. Wu, J.; Morris, J.K. The population prevalence of Down's Syndrome in England and Wales in 2011. Eur. J. Hum. Genet. 2013, 21, 1016-1019. [CrossRef] [PubMed]

3. Antonarakis, S.E.; Skotko, B.G.; Rafii, M.S.; Strydom, A.; Pape, S.E.; Bianchi, D.W.; Sherman, S.L.; Reeves, R.H. Down Syndrome. Nat. Rev. Dis. Prim. 2020, 6, 1-20. [CrossRef] [PubMed]

4. Jennum, P.; Riha, R.L. Epidemiology of Sleep Apnoea-Hypopnoea Syndrome and Sleep-Disordered Breathing. Eur. Respir. J. 2009, 33, 907-914. [CrossRef] [PubMed]

5. Fernandez, F.; Edgin, J.O. Poor sleep as a precursor to cognitive decline in Down Syndrome: A Hypothesis. J. Alzheimer Dis. Parkinsonism 2013, 3, 124.

6. Johnson, B.H.; Mitchell, R.F. Obstructive Sleep Apnea in children with Down Syndrome: Demographic, Clinical, and Polysomnographic Features. Otolaryngol. Neck Surg. 2019, 160, 150-157. [CrossRef]

7. Resta, O.; Barbaro, M.P.; Giliberti, T.; Caratozzolo, G.; Cagnazzo, M.G.; Scarpelli, F.; Nocerino, M.C. Sleep related breathing disorders in adults with Down syndrome. Downs Syndr. Res. Pract. 2003, 8, 115-119. [CrossRef]

8. Trois, M.S.; Capone, G.T.; Lutz, J.A.; Melendres, M.C.; Schwartz, A.R.; Collop, N.A.; Marcus, C.L. Obstructive Sleep Apnea in Adults with Down Syndrome. J. Clin. Sleep Med. 2009, 5, 317-323. [CrossRef]

9. Virji-Babul, N.; Eichmann, A.; Kisly, D.; Down, J.; Haslam, R.H. Use of health care guidelines in patients with Down Syndrome by family physicians across Canada. Paediatr. Child Health 2007, 12, 179-183. [CrossRef]

10. Ghiassi, R.; Murphy, K.; Cummin, A.R.; Partridge, M.R. Developing A Pictorial Epworth Sleepiness Scale. Thorax 2010, 66, 97-100. [CrossRef]

11. Mohr, C.; Tonge, B.; Einfeld, S.L. The Development of a new measure for the assessment of psychopathology in adults with intellectual disability. J. Intellect. Disabil. Res. 2005, 49, 469-480. [CrossRef] [PubMed]

12. Taffe, J.R.; Tonge, B.J.; Gray, K.M.; Einfeld, S.L. Extracting More Information from Behaviour Checklists by Using Components of Mean Based Scores. Int. J. Methods Psychiatr. Res. 2008, 17, 232-240. [CrossRef] [PubMed]

13. Skrbo, A.; Begović, B.; Skrbo, S. Classification of drugs using the ATC system (Anatomic, Therapeutic, Chemical Classification) and the latest changes. Med. Arh. 2004, 58, 138-141.

14. Fuhrman, C.; Fleury, B.; Nguyên, X.-L.; Delmas, M.-C. Symptoms of Sleep Apnea Syndrome: High Prevalence and Underdiagnosis in The French Population. Sleep Med. 2012, 13, 852-858. [CrossRef] [PubMed]

15. A Collop, N.; Anderson, W.M.; Boehlecke, B.; Claman, D.; Goldberg, R.; Gottlieb, D.J.; Hudgel, D.; Sateia, M.; Schwab, R. Clinical Guidelines for the Use of Unattended Portable Monitors in the Diagnosis of Obstructive Sleep Apnea in Adult Patients. J. Clin. Sleep Med. 2007, 3, 737-747. [PubMed]

16. Iber, C.; Ancoli-Israel, S.; Chesson, A.L.; Quan, S.F. The AASM Manual for the Scoring of Sleep and Associated Events: Rules, Terminology and Technical Specifications, 1st ed.; American Academy of Sleep Medicine: Westchester, IL, USA, 2007 ; p. 59.

17. World Health Organization. BMI classification. Pharmacotherapy. 2006, pp. 4-9. Available online: https://www.euro.who. int/en/health-topics / disease-prevention/nutrition/a-healthy-lifestyle/body-mass-index-bmi?source=post_page (accessed on 31 August 2021).

18. Masa, J.F.; Corral, J.; Pereira, R.; Duran-Cantolla, J.; Cabello, M.; Hernández-Blasco, L.; Monasterio, C.; Alonso, A.; Chiner, E.; Rubio, M.; et al. Effectiveness of Home Respiratory Polygraphy for the Diagnosis of Sleep Apnoea and Hypopnoea Syndrome. Thorax 2011, 66, 567-573. [CrossRef] [PubMed]

19. Andreou, G.; Galanopoulou, C.; Gourgoulianis, K.; Karapetsas, A.; Molyvdas, P. Cognitive Status in Down Syndrome Individuals with Sleep Disordered Breathing Deficits (SDB). Brain Cogn. 2002, 50, 145-149. [CrossRef]

20. Cody, K.A.; Piro-Gambetti, B.; Zammit, M.D.; Christian, B.T.; Handen, B.L.; Klunk, W.E.; Zaman, S.; Johnson, S.C.; Plante, D.T.; Hartley, S.L. Association of Sleep with Cognition and Beta Amyloid Accumulation in Adults with Down Syndrome. Neurobiol. Aging 2020, 93, 44-51. [CrossRef] [PubMed]

21. Shete, M.M.; Stocks, R.M.S.; Sebelik, M.E.; Schoumacher, R.A. Effects of Adeno-Tonsillectomy on Polysomnography Patterns in Down Syndrome Children with Obstructive Sleep Apnea: A Comparative Study with Children without Down Syndrome. Int. J. Pediatr. Otorhinolaryngol. 2010, 74, 241-244. [CrossRef] [PubMed]

22. Capone, G.T.; Aidikoff, J.M.; Taylor, K.; Rykiel, N. Adolescents and Young Adults with Down Syndrome Presenting to A Medical Clinic with Depression: Co-Morbid Obstructive Sleep Apnea. Am. J. Med Genet. Part A 2013, 161, 2188-2196. [CrossRef]

23. Pannucci, C.J.; Wilkins, E.G. Identifying and Avoiding Bias in Research. Plast. Reconstr. Surg. 2010, 126, 619-625. [CrossRef] [PubMed] 
24. Tennant, A.; Badley, E.M.; Sullivan, M. Investigating the Proxy Effect and The Saliency Principle in Household Based Postal Questionnaires. J. Epidemiol. Community Heal. 1991, 45, 312-316. [CrossRef] [PubMed]

25. Hill, E.A.; Sawatari, H.; Nishizaka, M.K.; Fairley, D.M.; Chishaki, A.; Funakoshi, K.; Riha, R.L.; Ando, S.-I. A Cross-Sectional Comparison of the Prevalence of Obstructive Sleep Apnea Symptoms in Adults with Down Syndrome in Scotland and Japan. Am. J. Intellect. Dev. Disabil. 2020, 125, 260-273. [CrossRef] [PubMed]

26. Escourrou, P.; Grote, L.; Penzel, T.; McNicholas, W.T.; Verbraecken, J.; Tkacova, R.; Riha, R.L.; Hedner, J. The Esada Study Group the Diagnostic Method Has A Strong Influence on Classification of Obstructive Sleep Apnea. J. Sleep Res. 2015, 24, 730-738. [CrossRef] [PubMed] 